\title{
UM Shaper Command Inputs for CRONE Control: Application on a DC Motor Bench
}

\author{
Rim Jallouli Khlif $\mathbb{D}^{1,2}$ Ahmed Abid $\mathbb{D}^{1},{ }^{1}$ Pierre Melchior $\mathbb{D}^{3},{ }^{3}$ and Nabil Derbel $\mathbb{D}^{1,4}$ \\ ${ }^{1}$ Laboratory of Control \& Energy Management, ENIS, University of Sfax, Sfax, Tunisia \\ ${ }^{2}$ Higher Institute of Computer Science and Multimedia of Sfax, University of Sfax, Sfax, Tunisia \\ ${ }^{3}$ Integration from Materials to Systems Lab (IMS-UMR 5218 CNRS), Université De Bordeaux-Bordeaux INP, Bordeaux, France \\ ${ }^{4}$ Digital Research Center of Sfax, Sfax, Tunisia
}

Correspondence should be addressed to Rim Jallouli Khlif; rim.jalloulikhlif@isims.usf.tn

Received 27 March 2021; Accepted 4 May 2021; Published 30 May 2021

Academic Editor: Abdellatif Ben Makhlouf

Copyright $\odot 2021$ Rim Jallouli Khlif et al. This is an open access article distributed under the Creative Commons Attribution License, which permits unrestricted use, distribution, and reproduction in any medium, provided the original work is properly cited.

\begin{abstract}
This study proposes an approach to synthesize a three-impulse sequence input shaper with a negative impulse, known as Unity Magnitude (UM) shaper. The corresponding analytic model has been already achieved for undamped and low-damped systems. In this paper, the analytic design of UM shaper is demonstrated for the generalized case of damped systems for both types: integer and fractional orders. Hence, the UM shaper model has been designed for second-order systems with damped dynamics, associating a graphical fitting and an analytical procedure; then, it has been extended to explicit fractional derivative systems. Moreover, the feasibility and the effectiveness of the proposed on-off profile prefilter applied on a second-generation controller have been substantiated by experimental results on an instrumented DC motor bench.
\end{abstract}

\section{Introduction}

Industrial motion systems require the suppression of the vibration to ensure mostly the stability and also the accuracy. Researchers and engineers have worked on command generation approaches to ensure such a purpose. Input shaping strategy constitutes a key issue for reducing residual vibrations in motion control systems, based on its robustness and effectiveness [1, 2]. Singer and Seering are the pioneers by proposing a practical input shaping scheme for flexible structures $[2,3]$. It is an open-loop control approach, implemented by convolving a train of impulses with any desired command profile, to generate the shaped reference command used to drive the system [1]. However, open-loop controllers are subject of external disturbances; thus, input shaping is used in conjunction with feedback control to ensure good efficiency [3]. The design of shapers is achieved by determining timing instants and amplitudes of the impulses, depending on the natural frequency and the damping ratio of the vibratory system, and by solving a set of constraint equations $[2,4]$.
Several works dealt with input shaping since its originality and simplicity to be implemented. By using a two-impulse set, the Zero Vibration (ZV) shaper cancels perfectly the output vibrations and eliminates the overshoot in case of linear systems $[5,6]$. Robustness is strengthened by adding derivative constraints, inducing a new impulse for each order: Zero Vibration Derivative (ZVD) and Zero Vibration Derivative Derivative (ZVDD) [6]. These different options have been already implemented on industrial systems [7] such as flexible robot manipulators, rotary cranes [3], and flexible beams.

Further, input shaping scheme has been extended to fit fractional order systems, notably for the ZV and ZVD shapers [8-12]. It has been associated with CRONE controllers for both real and complex orders, as well as fractional order PID controllers [13].

To overcome the delay induced by added impulses, new shapers have been designed by employing negative amplitudes that permit improving the rise time, shortening the shaper duration, and cancelling undesired oscillations [14-16]. However, unmodeled high modes can be subject of 
excitation [15]. Unity Magnitude (UM) shaper is one of these shapers as it allows negative impulses to be generated by switching impulses amplitudes between positive and negative unity [17-19]. Analytic definition of impulses timing is complex to solve for damped systems [14, 15, 17]. In [17], a restricted solution of UM shaper impulses times has been presented, only for second-order undamped systems and low-damped ones, with a damping ratio value limited between 0 and 0.35 .

The present paper deals basically with the design of the UM shaper for explicit fractional derivative order systems. For this purpose, the first challenge is to overcome the restriction on second-order damped systems. Thus an, indepth work has been undertaken to write UM shaper impulse time location models [18]. A graphical approach has been associated with such development. In a second step, the elaborated approach has been extended for the case of explicit fractional derivative systems, with the purpose to associate it with the CRONE control scheme. Thus, the developed analytic model of the UM shaper has been validated by the implementation on a DC motor test bench in association with a second-generation CRONE controller.

This paper is organized as follows. Section 2 is reserved to a background on the CRONE control and explicit fractional order systems. Section 3 introduces a background on the Unity Magnitude shaper. In Section 4, the problem description and the main contribution of this work are presented. Section 5 details the analytical synthesis of the UM shaper for second-order damped systems. Section 6 deals with the extension of the new analytic solution to explicit fractional derivative systems. In Section 7, real experimentation on a test bench with a CRONE controller is run in order to validate the effectiveness of the proposed approach. Finally, this paper ends with a conclusion.

\section{Background on Second-Generation Crone Control and Explicit Fractional Order Systems}

Fractional order robust control is named CRONE control by reference to the French nomination "Commande Robuste d'Ordre Non Entier." The CRONE Control System Design (CSD) methodology has been introduced since the 1980s by Oustaloup $[20,21]$ and is basically considered as a frequency-domain method. It relies on the unity-feedback control theory. Three CRONE CSD methods have been established and applied in several application fields. The main idea among the synthesis of these three approaches is to set the controller or the open-loop transfer function with reduced number of parameters and to define it by a fractional order integrodifferentiation [22, 23]. Thus, the major advantage of the CRONE controller, mainly its robustness, is due to its few number of parameters.

The second-generation CRONE controller defines the open-loop transfer function in a limited frequency interval around the cross-over frequency $\omega_{c g}$ as $n$ fractional order integrator (equation (1)), $n \in \mathbb{R}$, and $n$ is limited to ]1, 2 [ to ensure stability:

$$
\beta(s)=\left(\frac{1}{\tau s}\right)^{n},
$$

where $\tau=\left(1 / \omega_{c g}\right)$.

In this interval range, the Nichols locus of $\beta(j \omega)$ is a vertical straight line, called frequency template, whose phase position is strictly related to the noninteger order $n$. As the plant parameter $\left(\omega_{c g}\right)$ varies, the template slides vertically keeping the same phase location. Thus, this vertical displacement allows the robustness in phase margin, resonant peak, modulus margin, and damping ratio of the closed-loop system [22, 23].

In closed loop, a second-generation CRONE controller is described by a fundamental transfer function, which refers to explicit fractional derivative systems and is given by

$$
T(s)=\frac{1}{1+(\tau s)^{n}},
$$

where $\tau$ is called time constant and $n \in \mathbb{R}$ is the order of the system. Obviously, works and findings corresponding to explicit fractional derivative systems are valuable for secondgeneration controllers once synthesized according to desired performances.

\section{Background on Unity Magnitude Command Inputs}

Classical shapers, called Zero Vibration (ZV), are based on convolving two positive impulses to any command signal so that output vibrations are cancelled [2] as shown in Figure 1.

Such a preshaping technique adds a delay to the plant response in comparison to its natural dynamic. Consequently, in order to improve the rise time and shorten the shaper duration, negative impulse amplitudes are introduced as in $[15,24]$. The UM shaper is the case where amplitudes are required to switch between +1 and -1 as illustrated in Figure 2 [15]. Thus, the UM shaped command can be easily generated using on-off actuations differently from the ZV shaped input profile (Figure 1) [24].

In general, for a second-order system with a natural frequency $\omega_{n}$ and a damping ratio $\xi$, an input shaper is determined by satisfying residual vibration constraint equation (3) $[1,2,5,17]$.

$$
\left\{\begin{array}{l}
C\left(\omega_{n}, \xi\right)=\sum_{k=1}^{N} A_{k} e^{-\xi \omega_{n}\left(t_{N}-t_{k}\right)} \cos \left(t_{k} \omega_{n} \sqrt{1-\xi^{2}}\right)=0, \\
S\left(\omega_{n}, \xi\right)=\sum_{k=1}^{N} A_{k} e^{-\xi \omega_{n}\left(t_{N}-t_{k}\right)} \sin \left(t_{k} \omega_{n} \sqrt{1-\xi^{2}}\right)=0 .
\end{array}\right.
$$

$A_{i}$ and $t_{i}$ are, respectively, the amplitude and the location time of the $i^{\text {th }}$ impulse and $N$ is the number of impulses of the shaper. Equation (4) is considered so that the shaped command produces the same rigid body motion as the unshaped input command.

$$
A_{1}+A_{2}+A_{3}=1 \text {. }
$$




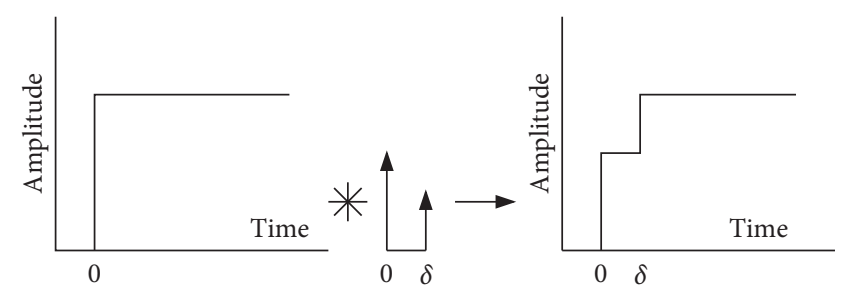

FIGURE 1: Input shaping principle.

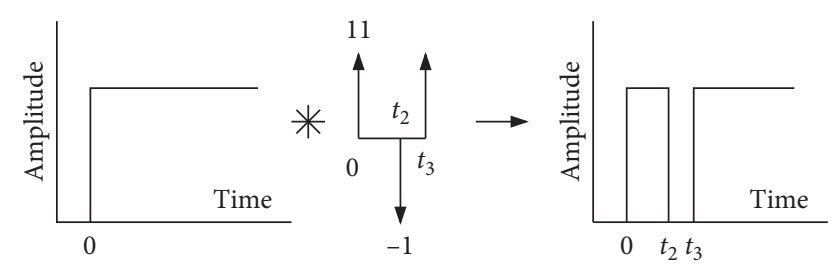

FIGURE 2: UM command shaper.

Considering the following system (5) dealing with the on-off amplitude profile, UM shaper needs to be identified through the location times of its three impulses. The first one is intuitively made at $t_{1}=0 \mathrm{~s}$ to ensure optimal shaper duration.

$$
\left\{\begin{array}{l}
A_{1}=1 \\
A_{2}=-1 \\
A_{3}=1
\end{array}\right.
$$

Now, substituting into equation (3), it becomes

$$
\left\{\begin{array}{l}
1-e^{\xi \omega_{n} t_{2}} \cos \left(\sqrt{1-\xi^{2}} \omega_{n} t_{2}\right)+e^{\xi \omega_{n} t_{3}} \cos \left(\sqrt{1-\xi^{2}} \omega_{n} t_{3}\right)=0, \\
-e^{\xi \omega_{n} t_{2}} \sin \left(\sqrt{1-\xi^{2}} \omega_{n} t_{2}\right)+e^{\xi \omega_{n} t_{3}} \sin \left(\sqrt{1-\xi^{2}} \omega_{n} t_{3}\right)=0 .
\end{array}\right.
$$

However, solving analytically equation (6) is likely to be difficult and needs linear and nonlinear approximations $[17,18]$. Though, the proposed solution does not give exact results for all damping ratio values.

The solution for undamped systems is easy to be determined using the amplitude summation constraint, the zero vibration constraint, and a trigonometric identity, which lead to designing the UM shaper for undamped systems as follows [15]:

$$
\left[\begin{array}{l}
A_{i} \\
t_{i}
\end{array}\right]=\left[\begin{array}{ccc}
1 & -1 & 1 \\
0 & \frac{1}{\omega_{n}} \cos ^{-1}\left(\frac{1}{2}\right) & \frac{1}{\omega_{n}} \cos ^{-1}\left(-\frac{1}{2}\right)
\end{array}\right] .
$$

\section{Problem Description and Main Contribution}

Robust control and vibration cancellation constitute two potential objectives for a large variety of real problems coming from different fields of engineering sciences and branches of industries. In several solutions, both objectives have been solved separately, and even in combined solutions, some drawbacks remain. In fact, the fractional order controllers (CRONE controllers) and the input shaping approach are well known in robust control and vibration cancellation, respectively. Even when combined with ZV and ZVD shapers [8-12], the CRONE control loop induces a delayed system response. Besides, the delay is increased if the robustness is strengthened by means of the ZVD shaper.

The main contribution of this work consists of designing a control loop that ensures both objectives, combining the robustness and the simplicity of the implementation of a CRONE controller and the vibration reduction using the UM shaper, with the advantage of no delay time generation. This challenge is achieved following two steps:

(i) First, the analytic synthesis of the UM shaper for second-order damped systems

(ii) Second, the UM shaper design for explicit fractional derivative systems

Results show that, with the UM shaper, the desired objective is ensured: actuators are less solicited due to the vibration cancellation, and the system dynamic is fastened as no delay time is introduced. Moreover, real experimentation on a DC motor test bench is run to validate the effectiveness of the proposed control approach.

\section{Analytic Synthesis of UM Shaper for Damped Systems}

Unity Magnitude shaper has the key feature to be easily implemented and to provide reduced control duration that guarantees no response delay. Thus, it has been adopted in many application areas as material-handling, lifting, and positioning applications $[3,25,26]$. The big challenge is to succeed to prove an analytical solution for time locations of the UM shaper in case of damped systems, which is likely to be complex as mentioned in previous works [17]. However, Gürleyük in [17] has elaborated one solution which has the disadvantage to be limited to low values of damping ratios varying between 0 and 0.35 . In the sequel, analytic synthesis of UM shaper for second-order systems is successfully elaborated without any restriction on the damping ratio, which can largely vary from 0 to 1 . The work is based on graphical estimations that permit deducing a key relation between second and third impulses times; then, further analytical development is completed.

5.1. Graphical Solution for UM Impulses Times. As a first step, a graphical solution for times $t_{2}$ and $t_{3}$ has been found by using a curve fitting technique [17]. In fact, the idea is to fix $t_{2}$ randomly and then search for $t_{3}$ graphically, satisfying constraints of equation (6). An application example is used and is defined by a damping ratio and a natural frequency that are, respectively, given as $\xi=0.5$ and $\omega_{n}=10 \mathrm{rad}$. Time $t_{2}$ was minutely chosen equal to $0.2265 \mathrm{~s}$, and time value of $t_{3}$ satisfying the constraints is deduced graphically and $t_{3}=0.2564 \mathrm{~s}$. Consequently, the resulting UM shaper configuration is as follows: 


$$
\left[\begin{array}{c}
A_{i} \\
t_{i}
\end{array}\right]=\left[\begin{array}{ccc}
1 & -1 & 1 \\
0 & 0.2265 & 0.2564
\end{array}\right] .
$$

Figure 3 depicts the effect of the above synthesized UM shaper on the studied system.

Comparing both curves, the overshoot and the oscillations are perfectly cancelled for the shaped response regarding the unshaped one. Thus, the synthesized UM shaper by the curve fitting technique proves its effectiveness; nevertheless, advanced experimentation and studies should be achieved to formulate a generalized analytical solution. To ensure such a purpose, different simulations have been undertaken for varying damping ratio values. It derives an important characteristic: as the damping ratio values vary, impulses times $t_{2}$ and $t_{3}$ remain symmetrically positioned regarding the rise time $t_{r}$. Thus, the following property is written:

$$
t_{r}=\frac{t_{2}+t_{3}}{2}
$$

Property of equation (9) will be used next to express impulses times $t_{2}$ and $t_{3}$, for damped systems.

5.2. Analytic UM Shaper Command Profile. Basically, the analytic equation of a second-order system step response is written as

$$
y(t)=1-\frac{1}{\sqrt{1-\xi^{2}}} e^{-\xi \omega_{n} t} \sin \left(\omega_{n} \sqrt{1-\xi^{2}} t+\arctan \left(\frac{\sqrt{1-\xi^{2}}}{\xi}\right)\right) .
$$

Further, in the case of unity step command, the rise time $t_{r}$ is expressed by the following equation:

$$
t_{r}=\frac{1}{\omega_{n} \sqrt{1-\xi^{2}}}\left(-\arctan \left(\frac{\sqrt{1-\xi^{2}}}{\xi}\right)+\pi\right)
$$

By simply equaling equation (9) and equation (11), it leads to write that

$$
t_{3}=\frac{2}{\omega_{n} \sqrt{1-\xi^{2}}}\left(-\arctan \left(\frac{\sqrt{1-\xi^{2}}}{\xi}\right)+\pi\right)-t_{2}
$$

Even with equation (12), the resolution of equation (6) is still complex. Therefore, deeper graphical investigation is worth of conducting to reduce difficulties.

Let us consider that the interval of time separating the second from the third impulse is likely short; then, it can be assumed that the response of the system upon this range of time is perfectly fitted to the tangent at the middle time $t_{r}$. The curve slope at the instant $t_{r}$ is deduced as follows:

$$
y^{\prime}\left(t_{r}\right)=\omega_{n} \exp \left(\frac{\xi}{\sqrt{1-\xi^{2}}}\left(\arctan \left(\frac{\sqrt{1-\xi^{2}}}{\xi}\right)-\pi\right)\right) .
$$

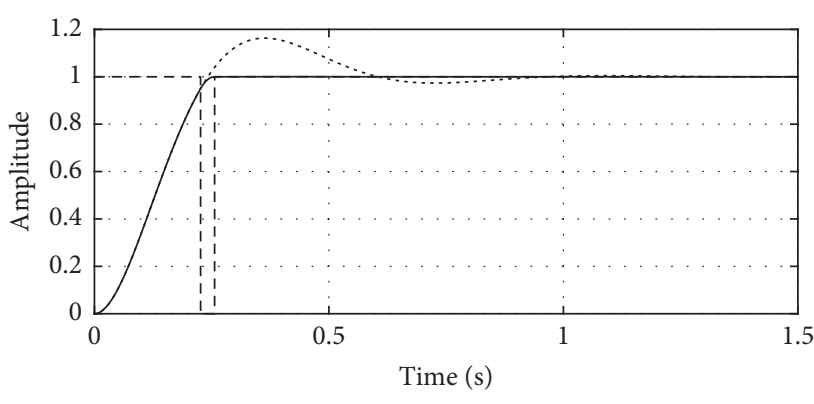

FIgURE 3: Responses of the studied system: unshaped case (...) and UM shaped case (-).

Moreover, by means of graphical approximation, the slope of the tangent at the point $t_{r}$ is identified as follows [18]:

$$
y^{\prime}\left(t_{r}\right)=\omega_{n}^{2}\left(t_{3}-t_{2}\right) .
$$

Bringing together equation (9) and equation (14), the second impulse time $t_{2}$ can be expressed as follows:

$$
t_{2}=t_{r}-\frac{1}{2 \omega_{n}^{2}} y^{\prime}\left(t_{r}\right)
$$

The third impulse time expression $t_{3}$ is also deduced as in equation (16):

$$
t_{3}=t_{r}+\frac{1}{2 \omega_{n}^{2}} y^{\prime}\left(t_{r}\right)
$$

Now, having expressions of equations (11), (13), (15), and (16), UM shaper is analytically designed as follows:

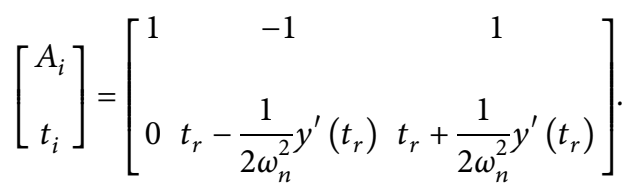

Validation of the graphical approximation used for the analytical development is proved through a set of systems with different damping ratios. A comparison between computed time values and estimated ones by curve fitting is established in Table 1. Relevant error values are also mentioned in Table 1. It shows that, for all the cases, the error is at least of $10^{-4}$ order; therefore, it can be neglected.

To conclude, the analytical approach of the UM shaper design for damped systems is well approved in the case of second-order systems without any restriction on damping ratio values, and UM shaper model is given by equation (17).

\section{UM Shaper Design for Explicit Fractional Derivative Systems}

In the previous section, the main contribution is the elaboration of an analytical model for UM shapers for secondorder damped systems. Now, the work deals with the extension of such an approach on explicit fractional derivative systems $[18,27]$ so that it can be applied for the case of second-generation CRONE control. 
TABLE 1: Comparison of both Um shaper design methods: graphical fitting and analytical calculus.

\begin{tabular}{lccc}
\hline & $\xi=0.2$ & $\xi=0.5$ & $\xi=0.7$ \\
\hline$t_{2 \mathrm{fit}}$ & 0.14445 & 0.2265 & 0.32346 \\
$t_{3 \mathrm{fit}}$ & 0.21560 & 0.25645 & 0.33350 \\
$t_{2 \mathrm{cal}}$ & 0.14597 & 0.2271 & 0.32350 \\
$t_{3 \mathrm{cal}}$ & 0.21562 & 0.2569 & 0.333505 \\
$\Delta t_{2}$ & 0.0006 & 0.00021 & 0.00004 \\
$\Delta t_{3}$ & 0.00045 & 0.00018 & 0.00000 \\
\hline
\end{tabular}

6.1. Response in Time Domain. In the operational field, a fundamental system is called explicit fractional derivative when its transfer function is described by equation (2) [21].
In the time domain, the step response of an explicit fractional system is written as mentioned in equation (18) $[21,28]$ :

$$
y(t)=\underbrace{1-\frac{2}{n} e^{(t / \tau) \cos (\pi / n)} \cos \left(\frac{t}{\tau} \sin \frac{\pi}{n}\right)}_{y_{\text {osc }}(t)} \underbrace{-\frac{\tau^{n} \sin (n \pi)}{\pi} \int_{0}^{+\infty} \frac{x^{n-1} e^{-x t}}{1+2(\tau x)^{2} \cos (n \pi)+(\tau x)^{2 n}} \mathrm{~d} x}_{y_{a p}(t)} .
$$

For practical reasons, equation (18) is divided into two modes: the aperiodic mode as in equation (19) and the oscillatory mode as in equation (20).

$$
y_{a p}(t)=-\frac{\tau^{n} \sin (n \pi)}{\pi} \int_{0}^{+\infty} \frac{x^{n-1} e^{-x t}}{1+2(\tau x)^{2} \cos (n \pi)+(\tau x)^{2 n}} \mathrm{~d} x,
$$

$y_{o s c}(t)=1-\frac{2}{n} e^{(t / \tau) \cos (\pi / n)} \cos \left(\frac{t}{\tau} \sin \frac{\pi}{n}\right)$

As the aperiodic mode goes towards zero rapidly, the oscillatory mode is dominant and used to write constraints of residual vibrations, robustness, impulse times, and amplitudes $[9,18,27]$. For this mode, the damping ratio is set by the fractional order $n$ and expressed as in equation (21).

$$
\xi(n)=-\cos \frac{\pi}{n}
$$

6.2. Extension of UM Shaper for Explicit Fractional Derivative Systems. In previous works, Poty et al. $[8,9]$ and JallouliKhlif et al. [11, 12, 27] have already extended the ZV and ZVD shapers to explicit fractional derivative systems for real order and complex order, respectively. In the present work, the UM shaper is extended to real order explicit fractional derivative systems [18, 27].

Based on system (3), explicit fractional system constraints used for the time impulse calculation are deduced as in (22), using only the oscillatory mode $[8,11]$. In the sequel, $\xi(n)$ is referred to by $\xi$, and $\omega_{c g}$ is referred to by $\omega$.

$$
\left\{\begin{array}{l}
\sum_{k=1}^{N} A_{k} e^{-\xi \omega\left(t_{N}-t_{k}\right)} \cos \left(\sqrt{1-\xi^{2}} \omega t_{k}+\frac{\pi}{n}\right)=0 \\
\sum_{k=1}^{N} A_{k} e^{-\xi \omega\left(t_{N}-t_{k}\right)} \sin \left(\sqrt{1-\xi^{2}} \omega t_{k}+\frac{\pi}{n}\right)=0
\end{array}\right.
$$

In the case of UM shaper (on-off profile), these constraints are upgraded as follows:

$$
\left\{\begin{array}{l}
-\xi e^{-\xi \omega t_{3}}-e^{-\xi \omega\left(t_{3}-t_{2}\right)} \cos \left(\sqrt{1-\xi^{2}} \omega t_{2}+\frac{\pi}{n}\right)+\cos \left(\sqrt{1-\xi^{2}} \omega t_{3}+\frac{\pi}{n}\right)=0 \\
\sqrt{1-\xi^{2}} e^{-\xi \omega t_{3}}-e^{-\xi \omega\left(t_{3}-t_{2}\right)} \sin \left(\sqrt{1-\xi^{2}} \omega t_{2}+\frac{\pi}{n}\right)+\sin \left(\sqrt{1-\xi^{2}} \omega t_{3}+\frac{\pi}{n}\right)=0 .
\end{array}\right.
$$

Advanced analytic development undertaken in $[16,18]$ proved that both constraints of system (6) devoted to second-order systems and (23) dealing with explicit fractional order systems are equivalent. Thus, the analytical approach design of UM shaper detailed in Section (5) is extended to explicit fractional derivative order systems, and the solution is perfectly written as follows: 


$$
\left[\begin{array}{l}
A_{i} \\
t_{i}
\end{array}\right]=\left[\begin{array}{ccc}
1 & -1 & 1 \\
0 & \frac{1}{\omega}(\delta-\kappa) & \frac{1}{\omega}(\delta+\kappa)
\end{array}\right]
$$

with

$$
\begin{aligned}
& \delta=\frac{\theta}{\sqrt{1-\xi^{2}}}, \\
& \kappa=\frac{1}{2} e^{-\delta \xi}, \\
& \theta=\pi-\arctan \left(\frac{\sqrt{1-\xi^{2}}}{\xi}\right) .
\end{aligned}
$$

6.3. Simulation Results. Figures $4-6$ outline the UM shaper action hold on a set of explicit fractional derivative systems, for $n=1.5, n=1.7, n=1.9$, and $\tau=1 \mathrm{~s}$. Each figure presents both shaped and unshaped responses.

Results show a residual overshoot that still exists, unlike the case of a second-order system. This is related to the fact that only oscillatory mode is considered for calculations, which induces a nonperfect UM shaper action on the global response.

Nevertheless, comparing both responses of each figure (Figures 4-6) and dealing with performances values of Table 2, it can be depicted that the UM shaper enhances system performances in a considerable way as

(i) It allows an evident reduction of the overshoot especially for high values of order $n$

(ii) It improves the settling time

(iii) It respects the rising time for all the cases

Besides, oscillation cancellation is proved.

\section{UM Shaper for CRONE Control Experimentation on a DC Motor Bench}

7.1. Description of the Plant. The association of UM shaper with second-generation CRONE control is applied to the speed control of a DC motor testing bench which rotates a disk with 14 equal weights. The inertia $j_{m}$ driven by the motor depends on the number of mounted weights (Figure 7). This motor can be coupled to a second DC motor to simulate the disturbance torque, but in the present case, the motor is uncoupled [29].

The transmittance of the DC motor is given as follows:

$$
T(s)=\frac{\omega(s)}{V(s)}=\frac{K_{u}}{\left(1+\tau_{e} s\right)\left(f_{m}+j_{m} s\right)},
$$

and specifications are as mentioned below. $\mathrm{rad}^{-1}$

$K_{u}=2.34 \mathrm{~N} . \mathrm{m} . \mathrm{V}^{-1}, \tau_{e}=4.710^{-3} \mathrm{~s}, f_{m}=10^{-3}$ N.m.s.

Therefore, the transmittance of the motor bench is expressed by the following equation [29]:

$$
T(s)=\frac{2.34}{(1+0.0047 s)\left(0.001+j_{m} s\right)} .
$$

Parametric variation of the process depends on the number of used weights. Besides, the disc must be loaded symmetrically to have a regular distribution of the inertia. In the proposed application, three parametric states are considered:

(i) With minimum load (2 flyweights): $j_{m}=0.012 \mathrm{~kg} \cdot \mathrm{m}^{2}$

(ii) With $57 \%$ of the maximum load (8 flyweights): $j_{m}=0.06 \mathrm{~kg} \cdot \mathrm{m}^{2}$

(iii) At maximum load (14 flyweights): $j_{m}=0.096 \mathrm{~kg} \cdot \mathrm{m}^{2}$

7.2. Control Problem. The objective is to control the motor in spite of inertia variation and to guarantee:

(i) A static error equal to zero

(ii) No saturation of the actuators (linear mode) for a step variation of 50 turns. $\mathrm{s}^{-1}$

(iii) A response time of approximately $0.3 \mathrm{~s}$

(iv) A total reject of a step type control disturbance

7.3. Controller Synthesis. The synthesis of the second-generation controller is achieved by means of the module "CRONE Control System Design" from the Matlab Toolbox "Crone Toolbox" [30]. Details of the synthesis of the secondgeneration CRONE control are explained in [22, 23, 30].

The second-generation CRONE controller is synthesized and has a fractional order $n=1.77$. It is approximated by a rational transmittance, obtained by fitting its frequency response.

7.4. UM Shaper Design. In order to reduce the rate of oscillations and the overshoot of the response to a speed step, the motor is controlled through the UM shaper. The shaper synthesis is made for the three configurations of masses mentioned above and offers the following designs, respectively, for the minimum load, $57 \%$ of the maximum load, and maximum load:

$$
\begin{aligned}
& {\left[\begin{array}{l}
A_{i} \\
t_{i}
\end{array}\right]=\left[\begin{array}{ccc}
1 & -1 & 1 \\
0 & 0.0267 & 0.0393
\end{array}\right],} \\
& {\left[\begin{array}{c}
A_{i} \\
t_{i}
\end{array}\right]=\left[\begin{array}{ccc}
1 & -1 & 1 \\
0 & 0.0668 & 0.0982
\end{array}\right],} \\
& {\left[\begin{array}{c}
A_{i} \\
t_{i}
\end{array}\right]=\left[\begin{array}{ccc}
1 & -1 & 1 \\
0 & 0.0864 & 0.127
\end{array}\right] .}
\end{aligned}
$$

7.5. Results and Performances. Figures 8-10 present experimental results in speed for the three cases of masses configuration of the motor bench (maximum weight, 57\% of maximum weight, and minimum weight). A comparison between the classical CRONE control step response and a 


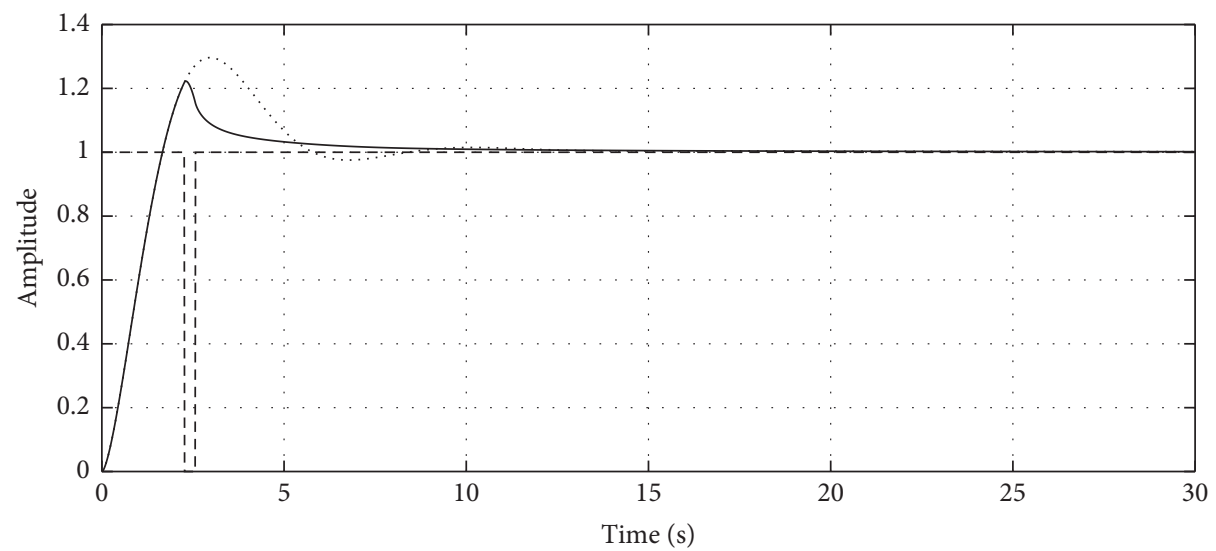

FIGURE 4: Responses of the studied system $(n=1.5)$ : unshaped case $(\ldots)$ and UM shaped case $(-)$.

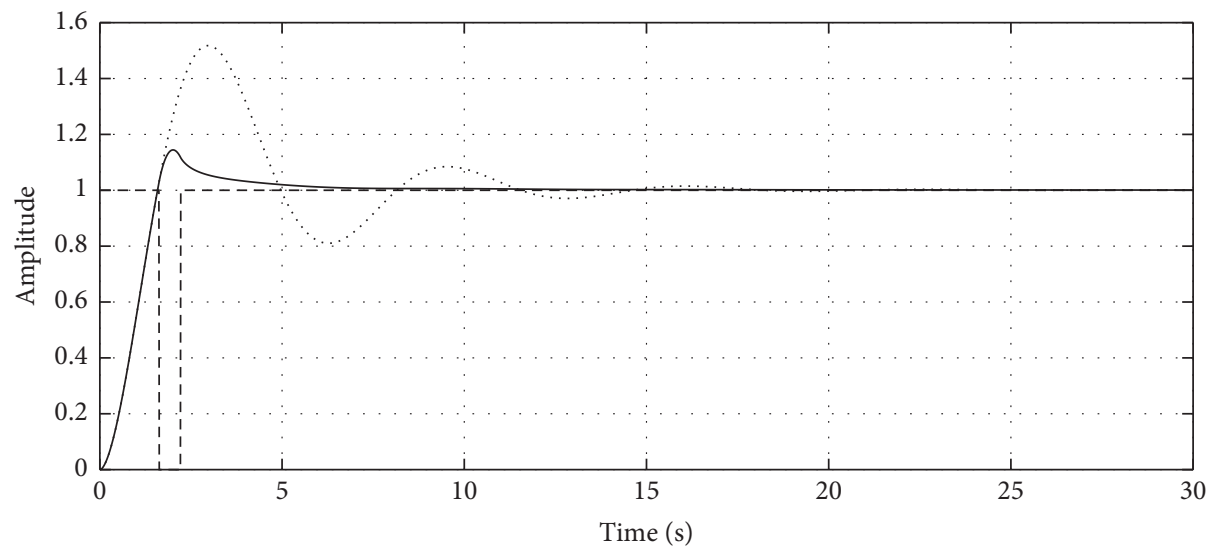

FIGURE 5: Responses of the studied system $(n=1.7)$ : unshaped case $(\ldots)$ and UM shaped case $(-)$.

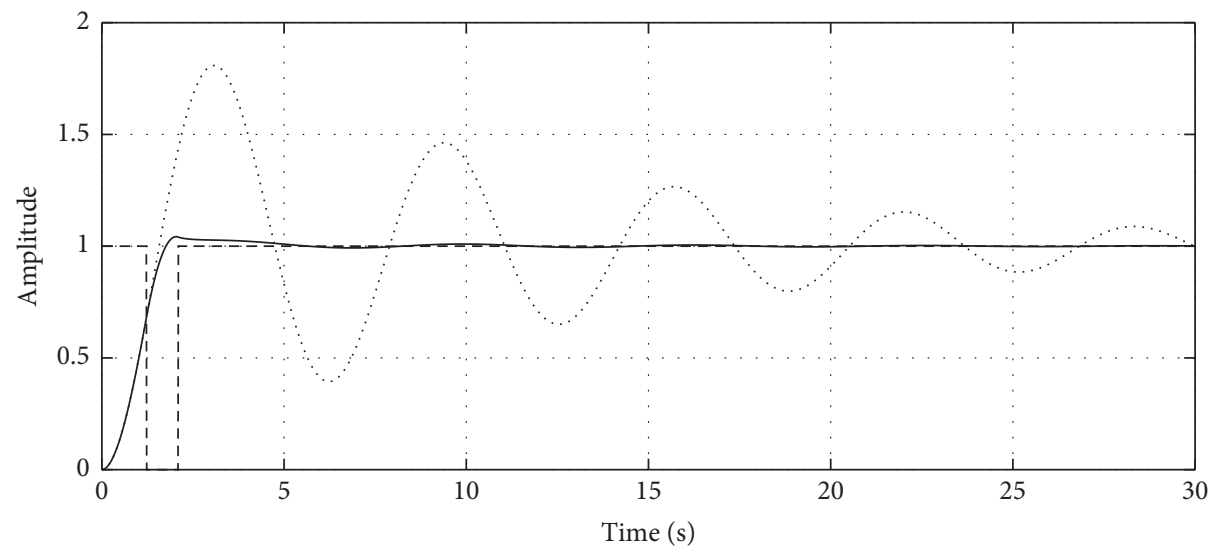

Figure 6: Responses of the studied system $(n=1.9)$ : unshaped case $(\ldots)$ and UM shaped case $(-)$.

CRONE control with UM shaper response is presented. Corresponding command signals are given in Figures 11-13.

The settling time for each experiment is as displayed in Table 3. Overshoot values are presented in Table 4.
According to Tables 3 and 4, control specifications are satisfied, and four advantages related to the use of the UM shaper are deduced:

(i) The settling time is reduced by a factor of 3 
TABLE 2: UM shaper performances.

\begin{tabular}{|c|c|c|c|c|}
\hline \multirow{2}{*}{ Fractional order } & \multicolumn{2}{|c|}{ Shaped response } & \multicolumn{2}{|c|}{ Unshaped response } \\
\hline & Overshoot & Settling time $(s)$ & Overshoot & Settling time $(s)$ \\
\hline$n=1.5$ & $22 \%$ & 3.9 & $30 \%$ & 5.16 \\
\hline$n=1.7$ & $14 \%$ & 3.12 & $52 \%$ & 10.6 \\
\hline$n=1.9$ & $4 \%$ & 1.62 & $80 \%$ & 34.8 \\
\hline
\end{tabular}

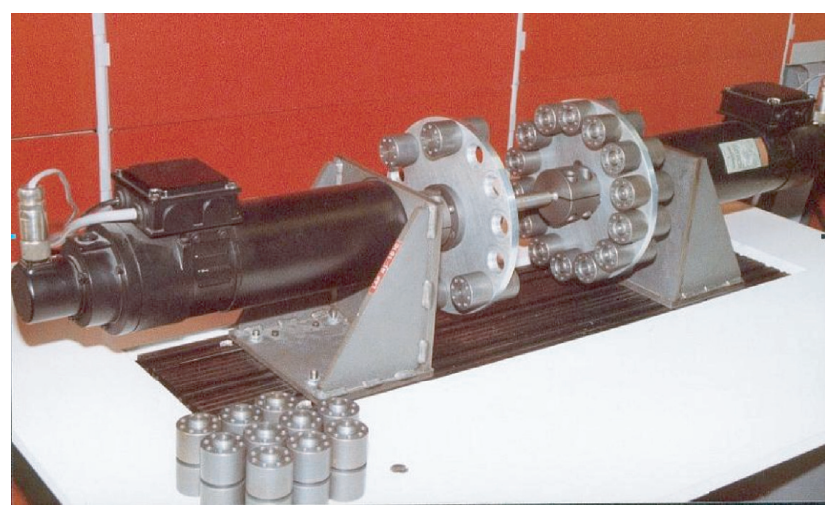

Figure 7: Instrumented test bench.

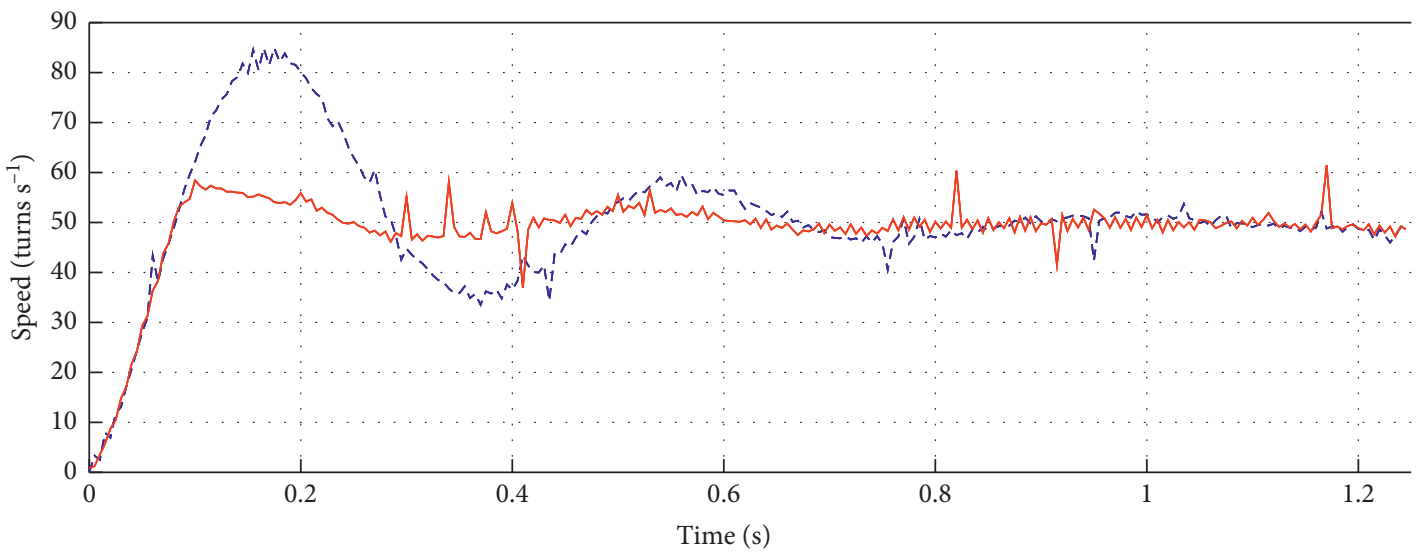

FIGURE 8: Response of the DC motor with maximum load (- -: unshaped, -: shaped).

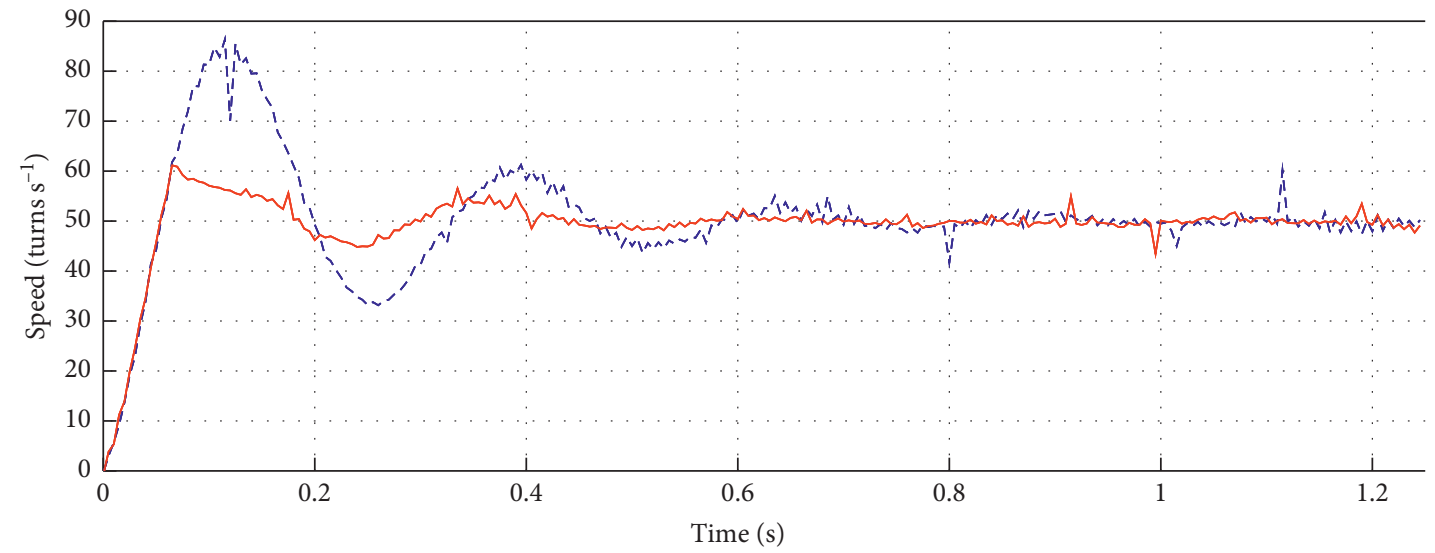

FIGURE 9: Response of the DC motor with 57\% of maximum load (- -: unshaped, - : shaped). 


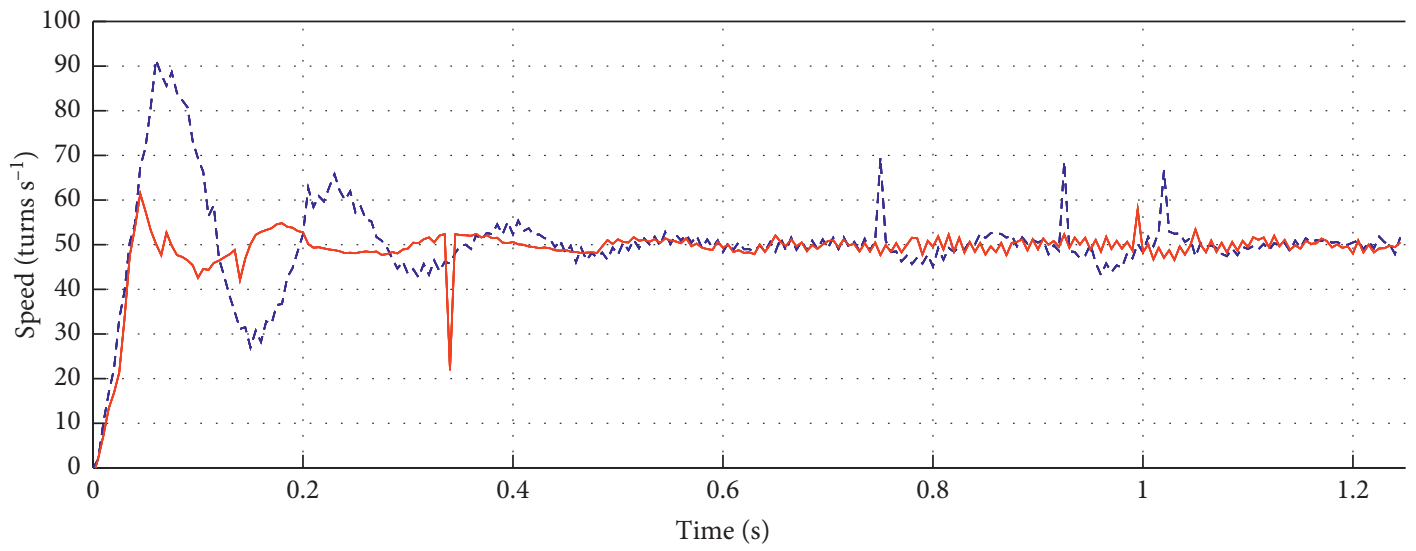

FIgURE 10: Response of the DC motor with minimum load (- -: unshaped, -: shaped).

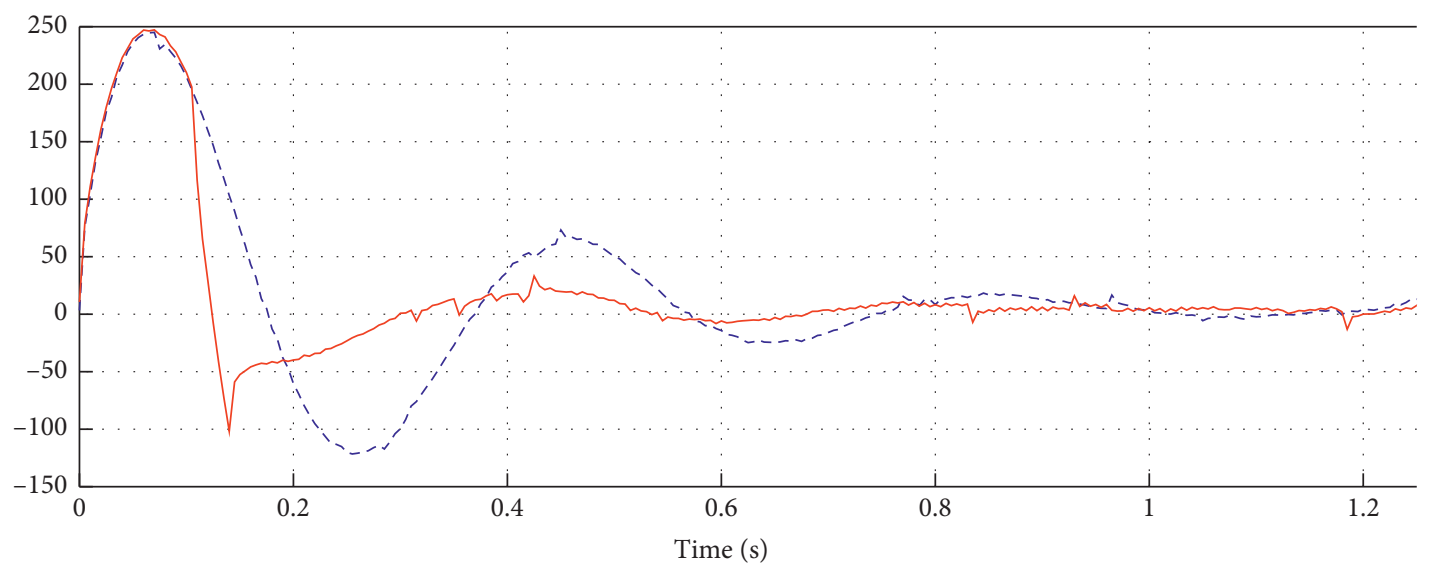

FIgURE 11: Control signal of the DC motor with maximum load (- -: unshaped, -: shaped).

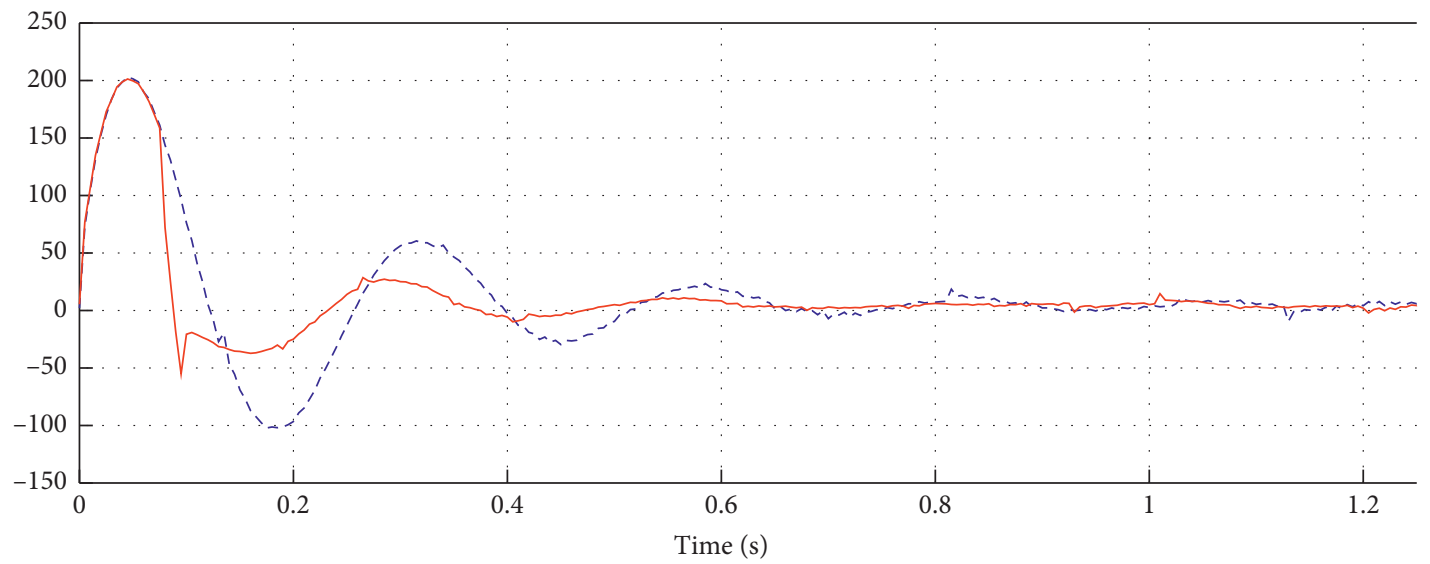

FIgure 12: Control signal of the DC motor with 57\% of maximum load (- -: unshaped, -: shaped). 


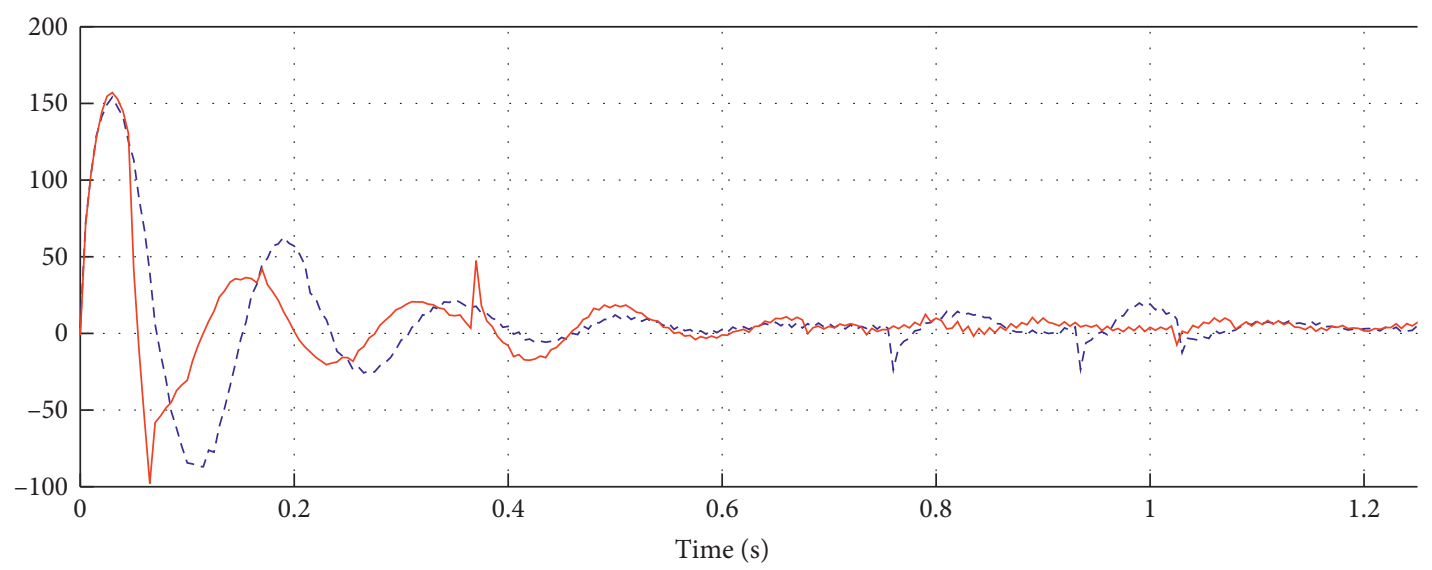

FIgURE 13: Control signal of the DC motor with minimum load (- -: unshaped, -: shaped).

TABle 3: Settling time.

\begin{tabular}{lcc}
\hline Settling time & Without UM shaper & With UM shaper \\
\hline Maximum load & $0.62 \mathrm{~s}$ & $0.2 \mathrm{~s}$ \\
57\% of maximum load & $0.44 \mathrm{~s}$ & $0.15 \mathrm{~s}$ \\
Minimum load & $0.45 \mathrm{~s}$ & $0.18 \mathrm{~s}$ \\
\hline
\end{tabular}

TABLE 4: Overshoot values.

\begin{tabular}{lcc}
\hline Overshoot & Without UM shaper & With UM shaper \\
\hline Maximum load & $70 \%$ & $19.8 \%$ \\
$57 \%$ of maximum load & $70 \%$ & $20.5 \%$ \\
Minimum load & $75 \%$ & $21 \%$ \\
\hline
\end{tabular}

(ii) The maximum overshoot is significantly reduced by a factor of 3.5

(iii) From a control point of view, the actuator is less stressed, which reduces the risk of saturation

(iv) Overshoots in all shaped cases are very close which proves the robustness of the UM shaper design approach

\section{Conclusion}

This work dealt with the synthesis of a three-impulse sequence input shaper known as Unity Magnitude (UM) shaper. It has the property to generate an on-off signal profile that permits cancelling undesired oscillations as well as improving the rise time and shortening the shaper duration in comparison with $\mathrm{ZV}$ and ZVD shapers. The main contribution has been achieved in two steps. First, the analytic design of the UM shaper was detailed for the case of damped second-order systems. Second, it has been generalized for explicit fractional order systems. The proposed approach associates graphical fitting and analytical procedure. Simulation results have proved good performances of the proposed model of the UM shaper. Feasibility and effectiveness are also supported by an experimental implementation on a DC motor bench commanded by a second-generation CRONE controller. It proves that the actuator is less stressed and oscillations are reduced.
As servicing has an important role in controlling robotic applications, this approach will be potentially applied on flexible robot arms with variable loads. In parallel, an indepth research work can be held on the generalized explicit derivative fractional order system response. It incorporates both aperiodic and oscillatory dynamics in order to ensure global controller design by the synthesis of a global response UM shaper.

\section{Data Availability}

Theoretical analysis and findings and experimental implementation parameters are the work result of the authors.

\section{Disclosure}

A part of the theoretical analysis was presented in $201916^{\text {th }}$ International Multi-Conference on Systems Signals and Devices (SSD). The implementation on the instrumental DC motor bench has never been published yet.

\section{Conflicts of Interest}

The authors declare that they have no conflicts of interest.

\section{Acknowledgments}

This work was achieved within the context of a research thesis on "Robust path tracking by input shaping in association with fractional order systems," in collaboration between Laboratory of Control \& Energy Management (CEM Lab), University of Sfax, Tunisia, and Integration from Materials to Systems Laboratory (IMS-UMR 5218 CNRS), Université de Bordeaux--Bordeaux INP, Bordeaux, France. The instrumental DC motor bench belongs to the CRONE team of IMS Laboratory. The authors would like to acknowledge the IMS Laboratory and particularly the CRONE team for supporting this work. 


\section{References}

[1] N. C. Singer, "Residual vibration reduction in computer controlled machines," Thesis, Massachussetts Institue of Technology, Cambridge, MA, USA, 1989.

[2] N. C. Singer and W. P. Seering, "Preshaping command inputs to reduce system vibration," Journal of Dynamic Systems, Measurement, and Control, vol. 112, no. 1, pp. 76-82, 1990.

[3] A. Alhassan, Z. Mohamed, A. M. Abdullahi, A. A. Bature, A. Haruna, and N. M. Tahir, "Input shaping techniques for sway control of a rotary crane system," Jurnal Teknologi, vol. 80 , no. $1,2018$.

[4] C. G. Kang, "Impulse vectors for input-shaping control: a mathematical tool to design and analyze input shapers," IEEE Control Systems Magazine, vol. 39, no. 4, pp. 40-55, 2019.

[5] W. Singhose, W. Seering, and N. Singer, "Residual vibration reduction using vector diagrams to generate shaped inputs," Journal of Mechanical Design, vol. 116, no. 2, pp. 654-659, 1994.

[6] W. E. Singhose, W. P. Seering, and N. C. Singer, "Input shaping for vibration reduction with specified insensitivity to modeling errors," in Proceedings of the Japan-USA Symposium on Flexible Automation, Shimizu, Japan, July 1996.

[7] P. Zhao, Y. Zhou, and R. Zhou, "A new trajectory optimizing method using input shaping principles," Shock and Vibration, vol. 2018, Article ID 4173253, 11 pages, 2018.

[8] A. Poty, P. Melchior, F. Levron, B. Orsoni, and A. Oustaloup, "ZV and ZVD shapers for explicit fractional derivative systems," in Proceedings of the 11th International Conference on Advnced Robotics, Coimbra, Portugal, July 2003.

[9] A. Poty, P. Melchior, and A. Oustaloup, "Preshaping command inputs for explicit fractional derivative systems: application to CRONE control," in Proceedings of the 7th IEEE European Control Conference (ECC'03), Cambridge, UK, September 2003.

[10] R. Jallouli-Khlif, P. Melchior, N. Derbel, and A. Oustaloup, "Preshaping command inputs for third generation CRONE control: robustness study," in Proceedings of the Sixth IEEE Int. Multi-Conference on Systems, Signals and Devices (SSD’09), pp. 23-26, Djerba, Tunisia, March 2009.

[11] R. Jallouli-Khlif, P. Melchior, F. Levron, N. Derbel, and A. Oustaloup, "Analytical impulse response of third generation Crone control," in New Trends in Nanotechnology and Fractional Calculus Applications, pp. 343-356, SpringerVerlag, Berlin, Germany, 2010.

[12] R. Jallouli-Khlif, P. Melchior, N. Derbel, and A. Oustaloup, "Preshaping command inputs for third generation CRONE control: simulation on a DC motor bench," in Proceedings of the 5th Workshop on Fractional Differentiation and its Applications, Nanjing, China, May 2012.

[13] J. Zhang and Q. Huang, "Study on the control method of mine-used bolter manipulator based on fractional order algorithm and input shaping technology," Mathematical Problems in Engineering, vol. 2018, Article ID 3707359, 8 pages, 2018.

[14] B. W. Rappole Jr., N. C. Singer, and W. P. Seering, "Input shaping with negative sequences for reducing vibrations in flexible structures," in Proceeding in the American Control Conference, San Francisco, CA, USA, June 1983.

[15] W. Singhose, E. O. Biediger, Y. Chen, and B. Mills, "Reference command shaping using Specified-Negative-Amplitude input shapers for vibration reduction," Journal of Dynamic Systems, Measurement and Control, vol. 126, 2004.
[16] A. Abid, R. Jallouli-Khlif, P. Melchior, and N. Derbel, "Negative input shapers for explicit fractional derivative systems," in Proceedings of the IEEE International Conference on Modelling, Identification and Control, Sousse, Tunisia, December 2015.

[17] S. S. Gürleyük, "Optimal unity-magnitude input shaper duration analysis," Archive of Applied Mechanics, vol. 77, pp. 63-71, 2007.

[18] A. Abid, R. Jallouli-Khlif, P. Melchior, and N. Derbel, "Synthesis of unity magnitude shaper: extension for explicit fractional derivative systems," in Proceedings of the International Multi-Conference on Systems, Signals and Devices, Leipzig, Germany, March 2016.

[19] A. Abid, R. Jallouli-Khlif, P. Melchior, and N. Derbel, "Real time synthesis of UM shaper: extension on explicit fractional derivative systems," in Proceedings of the International MultiConference on Systems, Signals and Devices, Leipzig, Germany, March 2016.

[20] A. Oustaloup, The CRONE Control, European Control Conf. ECC'91, Grenoble, France, 1991.

[21] A. Oustaloup and B. Mathieu, La commande CRONE: $d u$ scalaire au multivariable, Hermes Editions, Paris, 1999.

[22] P. Lanusse, R. Malti, and P. Melchior, "Crone control-system design toolbox for the control engineering community: tutorial and case study," Philosophical Transactions of the Royal Society A Mathematical, Physical and Engineering Sciences, vol. 371, 2013.

[23] P. Lanusse, J. Sabatier, and A. Oustaloup, "Fractional order PID and first generation CRONE control system design: fractional order differentiation and robust control design," Intelligent Systems, Control and Automation: Science and Engineering, vol. 77, 2015.

[24] M. J. Robertson and W. E. Singhose, "Closed-form deflection limiting commands," in Proceeding of 2005 American Control Conference, Portland, OR, USA, June 2005.

[25] E. Maleki, F. Bazerghi, and W. Singhose, "Shaping commands to maximize pendulum swing," in Proceedings of the 19th International Congress on Sound and Vibration, Vilnius, Lithuania, July 2012.

[26] E. Maleki, W. Singhose, and S. S. Gürleyük, "Increasing crane payload swing by shaping human operator commands," IEEE Transactions on Human-Machine Systems, vol. 44, no. 1, 2014.

[27] R. Jallouli-Khlif, A. Abid, P. Melchior, and N. Derbel, "UM shaper for fractional order systems: time analysis and robustness study," in Proceedings of the 2019 16th International Multi-Conference on Systems, Signals and Devices (SSD), Istanbul, Turkey, March 2019.

[28] P. Bania, J. Baranowski, and M. Zagórowska, "Convergence of Laguerre impulse response approximation for noninteger order systems," Mathematical Problems in Engineering, vol. 2016, Article ID 9258437, 13 pages, 2016.

[29] P. Melchior, A. Poty, B. Orsoni, and A. Oustaloup, "Preshaping command inputs for 2 nd generation CRONE control: application on an instrumented DC motor bench," in Proceedings of DETC'03 ASME 2003 Design Engineering Technical Conferences and Computers and Information in Engineering Conference, Chicago, IL, USA, September 2003.

[30] P. Melchior, P. Lanusse, O. Cois, F. Dancla, and A. Oustaloup, "Crone toolbox for matlab: fractional systems toolbox. Tutorial workshop on fractional calculus applications in automatic control and robotics," in Proceedings of the 41st IEEE Conference on Decision and Control (CDC'02), Las Vegas, NV, USA, December 2002. 